\title{
Forscherinitiierte Studie aus Deutschland zur IgA-Nephropathie Publikation im „New England Journal of Medicine“
}

Seit über 50 Jahren wird in der Glomerulonephritisbehandlung eine entzündungshemmende, immunsuppressive Therapie (mit Kortikosteroiden, aber auch mit neueren Immunsuppressiva und Zytostatika) eingesetzt. Wie bei jeder schweren und/oder chronischen Erkrankung ist eine gute unterstützende Behandlung aller Begleitumstände (supportive Therapie) wichtig. Dazu gehört eine konsequente Blutdrucksenkung und Proteinuriebehandlung mit ACE-Inhibitoren (ACE: Angiotensin Converting Enzyme) und Angiotensin-Rezeptor-Antagonisten. Einzelne kleinere Studien hatten in der Vergangenheit Hinweise darauf gegeben, dass durch die antihypertensive und antiproteinurische Therapie ein ähnliches Resultat wie mit der immunsuppressiven Therapie erzielt werden könne.

Um das prospektiv-randomisiert zu überprüfen, wurde 2006 die STOP-IgAN'-Studie auf den Weg gebracht. Es handelt sich dabei um eine forscherinitiierte (,investor initiated“), industrieunabhängige Studie aus Deutschland, gefördert durch das Bundesministerium für Bildung und Forschung (BMBF), die unter Leitung Aachener Nephrologen (Thomas Rauen, Frank Eitner, Jürgen Floege) und Medizin-Statistikern (Christina Fitzner, Ralf-Dieter Hilgers) durchgeführt wurde. Sie ist Anfang Dezember 2015 im „New England Journal of Medicine“, einem der weltweit renommiertesten internationalen Journals, erschienen.

\section{Ergebnisse}

In der Studie erfolgte zunächst eine 6-monatige Run-in-Phase, in der alle Patienten nach einem intensivierten supportiven Protokoll behandelt wurden und die 309 von insgesamt 379 gescreenten

\begin{tabular}{l}
\hline Deutsche Gesellschaft \\
\hline für Nephrologie \\
Geschäftsführer DGfN: RA Tilo Hejhal \\
Geschäftsstelle DGfN: Uwe Stelbrink \\
Stefanie Sahr, Seumestr. 8, 10245 Berlin \\
Tel.: +49 30/5213-7269, Fax: +49 30/5213-7270 \\
E-Mail: gs@dgfn.eu, Internet: www.dgfn.eu \\
Vorstand der DGfN: \\
- Prof. Dr. Jürgen Floege, Aachen (Präsident) \\
- Prof. Dr. Kerstin Amann, Erlangen \\
- Prof. Dr. Mark-Dominik Alscher, Stuttgart \\
- Prof. Dr. Andreas Kribben, Essen \\
- Dr. Thomas Weinreich, Villingen-Schwennin- \\
gen
\end{tabular}

Patienten beendeten. Wie sich zeigte, konnte in dieser Phase bereits bei knapp $30 \%$ der Betroffenen die Proteinurie auf unter $0,75 \mathrm{~g} / \mathrm{d}$ gesenkt werden, also eine Krankheitsremission erreicht werden, sodass die Patienten nicht mehr den Einschlusskriterien entsprachen.

Die verbliebenen 162 Patienten wurden dann randomisiert, 80 erhielten weiterhin die intensivierte supportive Therapie, 82 erhielten zusätzlich Immunsuppressiva. Im Ergebnis zeigte die 3-jährige Studie, dass es keinen signifikanten Unterschied hinsichtlich der Erkrankungsprogression (gemessen am Nierenfunktionsverlust) zwischen den Gruppen gab. In der mit Immunsuppressiva behandel- ten Gruppe traten dagegen signifikant mehr Nebenwirkungen, auch gefährliche Infektionen, auf; ein Patient verstarb an einer Sepsis.

\section{Fazit}

Laut den Autoren wird die Studie einen Paradigmenwechsel in der Therapie der IgA-Nephropathie einleiten: „Für den klinischen Alltag bedeutet das Studienergebnis, dass zunächst immer eine intensivierte supportive Therapie (antihypertensive und antiproteinurische Medikation) erfolgen sollte. Erst bei einem Nichtansprechen kann dann nach sorgfältiger Risikoanalyse bei einzelnen Patienten der Einsatz einer immunsuppressiven Therapie erwogen werden. Der flächendeckende, sofortige Einsatz von Immunsuppressiva ist bei diesem Krankheitsbild ab sofort obsolet“, erklärt Prof. Jürgen Floege, Universitätsklinikum Aachen.

Pressestelle der DGfN

\footnotetext{
${ }^{1}$ Supportive Versus Immunosuppressive Therapy for the Treatment Of Progressive IgA Nephropathy
}

\section{Besuch der „Initiative Nierentransplantation“ beim Bundesgesundheitsminister}

Anfang Dezember 2015 empfing Bundesgesundheitsminister Hermann Gröhe Vertreter der „Initiative Nierentransplantation“, namentlich Peter Gilmer, Vorsitzender des Bundesvorstands des Bundesverbands Niere e. V., und Prof. Jürgen Floege, Präsident der Deutschen Gesellschaft für Nephrologie (DGfN) (Abb. 1). Der Minister sicherte der Initiative seine Unterstützung zu, wie er auch über Facebook mitteilte.

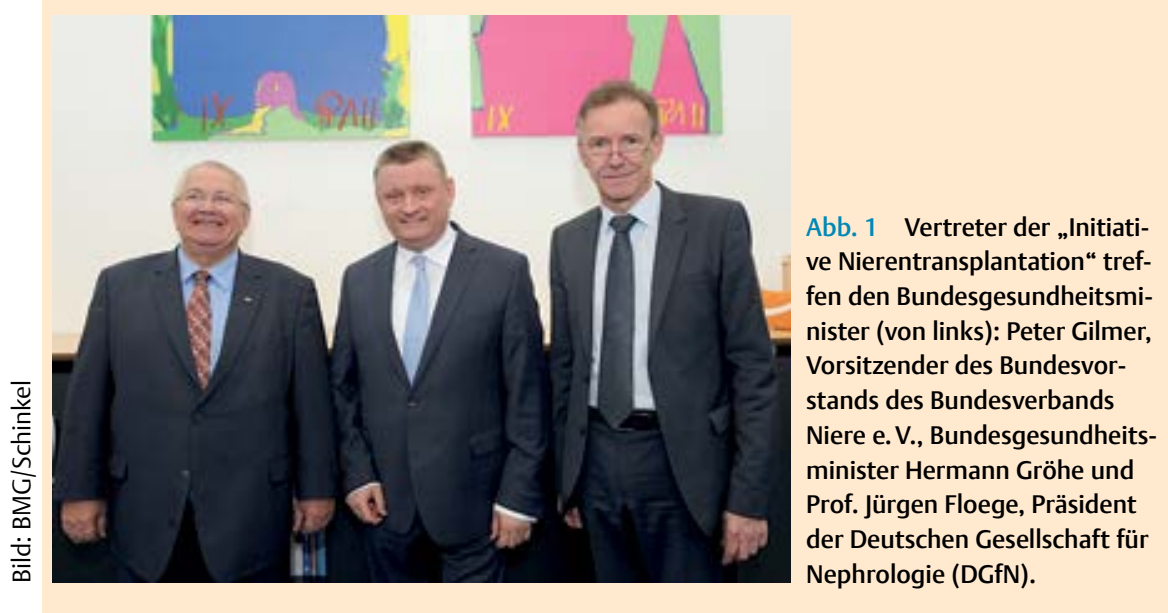

